\title{
Ontology Driven Continuous Learning Approach
}

\author{
Dakshi T. K. Geeganage and Asoka. S. Karunananda
}

\begin{abstract}
Learning is the act of acquiring new knowledge or updating the existing knowledge about different types of information. Human being starts the learning process with a minimal knowledge and then expands the knowledge by adding new information to the existing knowledge. Thus it is possible to model the learning process as ontology modelling process and the research has been conducted to develop an automated ontological solution for continuous learning. The solution demonstrates a system which can learn and expand the knowledge somewhat similar to a human being. The system consists of three major functions named as knowledge extraction, knowledge comparison and knowledge integration. This approach consists of two main research components. The ontology construction mechanism of the system was designed with over $60 \%$ of expected accuracy and knowledge integration can be evaluated over $75 \%$ accuracy. This system can be used as the main learning process of any application.
\end{abstract}

Index Terms-Knowledge, learning, ontology.

\section{INTRODUCTION}

Learning is the acquisition of knowledge or skills through study, experience, or being taught. Learning is about actively making sense of the world around us by taking in new information, comparing it to our current understanding and negotiating meaning out of those interactions. The human mind is better equipped to gather information about the world by operating within it than by reading about it, hearing lectures on it, or studying abstract models of it. Human being starts the learning process with a minimal knowledge, and then expands the knowledge by adding new information and making a structure by comparing the existing and new knowledge. Human being constructs his knowledge by adding totally new concepts or expanding the already existing content. People have identified the importance of machines which can take over human tasks and intelligent and advanced systems have become popular with the development of the technology. Most of the intelligent systems work by obtaining expert knowledge from different sources.

There's no automated methodology to gain and update existing knowledge somewhat similar to human knowledge acquisition. Knowledge acquisition and representation can be defined as crucial parts in learning.

Information available in different formats cannot be

Manuscript received October 10, 2014; revised January 16, 2015 Ontology Driven Continuous Learning Approach is a research conducted for the partial fulfilment of the requirements of the Degree of MSc in Artificial Intelligence.

Dakshi T. K. Geeganage was with the Universtiy of Moratuwa, Sri Lanka She is now with Sri Lanka Institute of Information Technology, Sri Lanka (e-mail: dakshi.g@sliit.lk).

Asoka S. Karunananda was with the University of Moratuwa, Sri Lanka He is now with Sir John Kotelawala Defence University, Sri Lanka (e-mail: asokakaru@yahoo.com). understood by a computer or a machine due to lack of a proper knowledge representation mechanism. It always requires more human effort in feeding the knowledge to the computers or the knowledgebase. Knowledge acquisition, knowledge representation and knowledge reasoning can be defined as key problems associated in automated AI systems. Knowledge acquisition task is difficult and involves many problems, this is why it is sometimes denoted as the 'knowledge acquisition bottleneck', as named by Feigenbaum [1]. This task is described as a bottleneck since it is very resource and time-consuming to elicit knowledge from experts, and experts often have a hard time expressing their knowledge explicitly, and some knowledge might not even be possible to express formally.

Several researches have been carried out in last two decades to simulate the human learning inside machines to make them intelligent. Eva Blomqvist [2] introduced a pattern based mechanism to construct ontology while Ekaterina Ovchinnikova and Kai-UweK"uhnberger [3] suggested a mechanism to generate ontology by comparing with key words. Giannis Varelas, Epimenidis Voutsakis and Paraskevi Raftopoulou [4] have invented a method to detect similarities between semantically similar documents. Their research was based on the WordNet. Paul Buitelaar, Daniel Olejnikand MichaelSintek [5] proposed a protégé plugin to automate the ontology creation process and Alexander Maedche and Steffen Staab [6] have presented a process of semi-automatic ontology learning from text. Mohamed Yehia Daha, Hesham A. Hassan and Ahmed Rafea [7] have presented an approach to generate ontology from text using semantic pattern-based approach. TextOntoEx [7] uses a semantic pattern based approach to convert text to ontology and it does not discover new relations other than the known contents. Most of the above mentioned efforts were semi-automated approaches and they have not focused on building a system which can learn automatically without human involvement.

Knowledge extraction and integration with the existing concepts are still under research. It is important to propose a common framework for knowledge representation that can be minimized the above mentioned problems for a certain extent. Ontologies have become the knowledge representation medium of choice in recent years for a range of computer science specialties including the Semantic Web, Agents, and Bio-informatics. In the context of knowledge sharing, Tom Gruber [8] uses the term ontology to mean a specification of a conceptualization.

Ontology is a description of the concepts and relationships that can exist for an agent or a community of agents. Ontological categories provide a means to classify all existing things, and the systematic organization of such categories allows to analyze the world that is made up by these things in a structured way [9]. Knowledge representation via ontology can be defined as a very successful mechanism to cover most 
of the objects and concepts in the real world.

There is a requirement of automating the learning process by constructing or modeling the ontology from the base level. If there is a way to construct and update the knowledge by integrating an ontology, it can be somewhat similar to the learning process of human beings. Human being constructs his basic knowledge and once he hear or learn new things, he will add them to the existing knowledge.

If there is a mechanism to update the knowledge in ontology by integrating new ontology, it will be useful for many applications. This process can be used as the main learning process of any application. Nevertheless still there is no successful mechanism to update and share knowledge by integrating two or more ontology. The research proposes a mechanism to update the existent knowledge by integrating new knowledge.

There are different learning strategies which are used by human beings to acquire and update their knowledge. Rote learning and meaningful learning can be defined as two main learning strategies. Rote learning is memorizing information so that it can be recalled by the learner exactly the way it was read or heard. The major technique used for rote learning is learning by repetition, based on the idea that a learner can recall the content. Meaningful learning is the concept that learned knowledge is fully understood to the extent that it relates to other knowledge. The system directly supports for both learning strategies when representing knowledge.

The aim of the research is to present an ontology based solution to automate the learning process of intelligent systems by constructing the knowledge from concepts and update the knowledge by expanding the existing content somewhat similar to a human being. Main research objectives are presenting ontology based solution to represent natural language concepts into meaningful ontology, development of a solution which starts the learning with minimal knowledge and eventually expands the knowledge without human involvement. Finally it is expected to automate the knowledge acquisition process of intelligent systems by embedding the Ontology Driven Continuous Learning Approach.

\section{ONTOLOGY IN LEARNING}

Ontology Driven Continuous Learning Approach is an application which is capable of representing knowledge similar to human knowledge representation. Human recognizes things by associating with known things and expand the knowledge. When learning new things the human being tries to identify the features, properties and relationships with other objects.

It is very difficult to identify important attributes, relationships among attributes, choosing the granularity of representation, representing sets of objects and finding the right structures to represent information.

Knowledge representation via ontology addresses all the problems of the knowledge representation. Ontology is a concept which can be used to model real word entities, their properties and relations among them in a taxonomic structure. The word "ontology" seems to generate a lot of controversy in discussions about Artificial Intelligence. It has a long history in philosophy, in which it refers to the subject of existence. It is also often confused with epistemology, which is about knowledge and knowing.

In the context of knowledge sharing, Tom Gruber [8] uses the term ontology to mean a specification of a conceptualization. Ontology is a description (like a formal specification of a program) of the concepts and relationships that can exist for an agent or a community of agents. This definition is consistent with the usage of ontology as set-of-concept-definitions, but more general. And it is certainly a different sense of the word than its use in philosophy. [10]

In the context of computer and information sciences, ontology defines a set of representational primitives with which to model a domain of knowledge or discourse. The representational primitives are typically classes (or sets), attributes (or data properties), and relationships (object properties or relations among class members. Ontologies are specifications of concepts and relations among them. They play a central role in semantic web applications by providing a shared knowledge about the objects in real world, which promotes reusability and interoperability among different modules. Therefore the quality of the ontology should be the first concern in any semantic application.

The key role of ontologies with respect to database systems is to specify a data modeling representation at a level of abstraction above specific database designs (logical or physical), so that data can be exported, translated, queried, and unified across independently developed systems and services. Successful applications to date include database interoperability, cross database search, and the integration of web services [11]. Ontologies have become a major tool for developing semantically rich applications. Ontologies can be used for many different purposes, as described by McGuinness [12]; to provide a controlled vocabulary, to customize and personalize search possibilities, to provide a structure from which to extend content, to perform word sense disambiguation, to provide interoperability support, and other similar tasks.

Learning can be seen as an ontological modeling process. Two different types of inputs can be provided to the system. The user can add a sentence or an already created ontology as input to the system. The system contains its existing knowledge about the area of Literature as the main ontology. The user can add a new concept or an ontology to the system. Once the system receives a new concept, the concept or sentence is divided into tokens. Then using the WordNet2.1 the system identifies the nouns, verbs, adjectives adverbs and synonyms for identified tokens. Then identified tokens will go through set of jape rules which valid with set of possible values. The system supports for rote learning strategy by matching with set of jape rules.

Then the system extracts the semantics of the new knowledge and tries to identify the content with known things.

If it couldn't identify with known things, then tokenized inputs will go through set of rules to learn things by going through the rules.

Thereafter system categorizes the concept into objects, relationships, properties etc... and the knowledge or concept will be converted into an ontology. If user adds an ontology the system goes through the owl file and identifies the 
available classes, instances, properties and relationships among different objects. Afterward the system is capable enough to find the existence of knowledge and identifies existing content and totally new concepts. Then the system compares the new knowledge with the existing knowledge available in main ontology by checking the classes, instances, data properties and object properties. The system updates the knowledge by going through three processes. Three processes will be lexical matching, semantic matching and rule based matching mechanisms. Initially, the system checks the new content by matching with the existing content (classes) by comparing strings or lexicons, then semantically checks the data properties and object properties and instances of classes. Wordnet 2.1 is used to identify the synonyms and similarities of the content. Thereafter formulated rules are fired on top of the content to check the accuracy and possibility of the occurrence. In this stage both rote learning and meaningful learning strategy will be accomplished by going through lexicons, semantics and rules. Finally necessary modifications and integrations to the main ontology are done by extracting new knowledge from a given ontology. If the new knowledge is partially available, update the main ontology by expanding the current content. If the knowledge is totally new or unavailable, the system represents the knowledge in appropriate way somewhat similar to human learning. Finally the main ontology will be expanded by absorbing necessary classes, sub classes, instances, data properties and object properties.

Main ontology will be updated with the new concepts and the output will be the updated main ontology (existing knowledge) with integrated new knowledge.

Fig. 1 elaborates the high level architecture of the system with the main modules and their functionality.

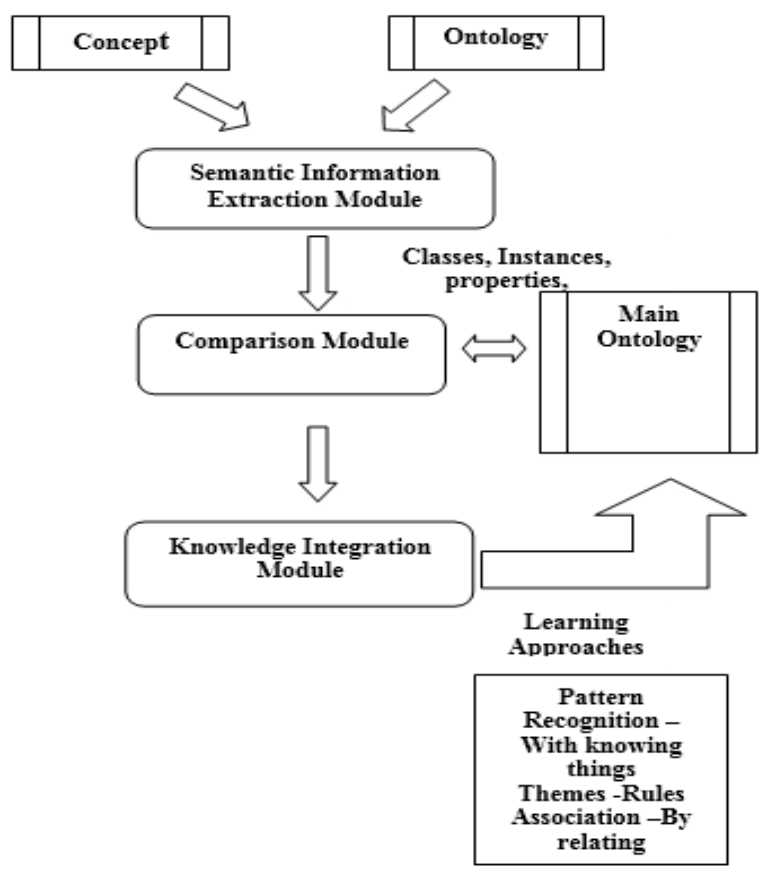

Fig. 1. High level architecture of the system.

\section{MethodolOGY}

Ontology Driven Continuous Learning Approach consists of three main modules and each module contains set of sub components to fulfill the process of each module.

The system has been developed using the java programming language and on top of the jdk1.6.0 environment. NetBeans 6.8 was used as the integrated development environment. Gate 5.1 API, Word Net-2.1, Protege 4.1 and OWL API were used in the implementation stage.

\section{A. Semantic Information Extraction Module}

Semantic information extraction module is responsible for extracting the semantics of a given content. When the system receives a concept (a sentence or a paragraph), semantic extraction module will break the concept into tokens and tries to identify the known things. Tokenization and named entity recognition are done using the Gate API. GATE is distributed with an Information Extraction system called ANNIE, A Nearly-New IE system.

The tokeniser splits the text into very simple tokens such as numbers, punctuation and words of different types. Then Jape rules are applied on top of the tokenized input for the purpose of pattern matching over annotation. JAPE is a language for doing regular-expression-style pattern matching over annotations rather than text. Using the JAPE rules the system can easily identify the known things and related things. The rote learning mechanism is accomplished with the aid of jape rules. Once the system identifies a particular token, it will be stored in an item list and it will be recalled from the list similar to recalling things from the memory.

Then the system identifies unknown tokens and passes them to the WordNet2.1 library. WordNet identifies nouns verbs and synonyms of the tokens. The module contains rules to recognize classes, properties, instances and relationships by analyzing the tokens. Finally, the module creates ontology by examining the classes, properties and relating them together.

Set of rules are defined to identify the components of ontology. Sample set of rules are given below;

- If the word is a noun given in the dictionary it is a class.

- If the word is a verb it is an object property between subject and object.

- If the word is an adjective it is a data property.

- After the adjective or verb and a preposition, if there is a numerical value, it is an integer data property.

Martin Wickramasinghe born in 19XX

Born is a data property with the value 19XX.

- After the adjective or verb and a preposition, if there is a string value, it is string data property.

Martin Wickramasinge lived in Koggala

Lives is a data property with the value Koggala

- If the word is a specific noun (proper noun) it can be an instance of particular class

E.g.: Madolduwa, Martin (Most probably they are not dictionary words)

- If the word is a pronoun (it, he, she, they) navigate to the previous sentence' subject noun.

If user adds an ontology, this module goes through the owl file and identifies the available classes, instances, properties and relationships among different objects.

Basically this module is responsible of extracting knowledge from an ontology or concept by considering the 
semantics of the components.

\section{B. Ontology Comparison Module}

The main ontology can be defined as the brain of the system and it contains the basic structure about the domain.

It is important to identify the existing knowledge before integrate the new knowledge to the main ontology. After the update process main ontology will be saved and in the comparison module, main ontology will be compared in all aspects (classes, properties, instances and relationships) by analyzing semantics over existing knowledge and new knowledge. Finally ontology comparison module displays new knowledge which is not available in the main ontology.

\section{Knowledge Integration Module}

Knowledge Integration module was developed by using Protege 4.1 and OWL API libraries. It is important to identify the nature of new knowledge before integrating it to the main ontology. Knowledge integration module applies different learning approaches before the integration process. WordNet2.1 library is used to identify the synonyms and similarities with existing content. Then the new content will be integrated to the main ontology by applying different learning approaches such as pattern recognition (With knowing things), themes (rules) and association (by relating).

\section{Knowledge Integration algorithm}

Input: Main owlfile maino, New owlfile newo

Output: Updated Ontology owlfile updatedo

Function Knowledge integration (owlfile maino, owlfile newo)

\{

String cls1,cls2, prop[];

owlfile updatedo=copy of owlfile maino;

cls1=MainClass(Main Ontology);

cls2=LeftMostLeafNode(New Ontology);

foreach Class cls 2 in owlfile newo

\{

FunctionWordAnalyzer(cls1.name, cls2.name)))

FunctionSemanticFinder(cls1.name, cls2.name);

FunctionSimilarityFinder(cls1,cls2,prop3[]);

The algorithm contains three sub algorithms to analyze words, finds meanings and to find the similarities. Knowledge integration algorithm works by considering two different aspects of ontology updating. First it does the lexical analysis and semantic analyses then finds the similarities and expands the knowledge tree. WordNet is embedded in the Semantic Analyzer function and it checks the synonyms of the words when it is considering the meanings of the concept. The brain of the system can be defined as the main ontology.

\section{Main ontology}
$<$ Declaration>
$<$ Class IRI="\#Author"/>
$</$ Declaration $>$
$<$ Declaration>
$<$ Class IRI="\#Book"/>
$<$ /Declaration $>$
$<$ Declaration $>$
$<$ Class IRI="\#Fictions"/>

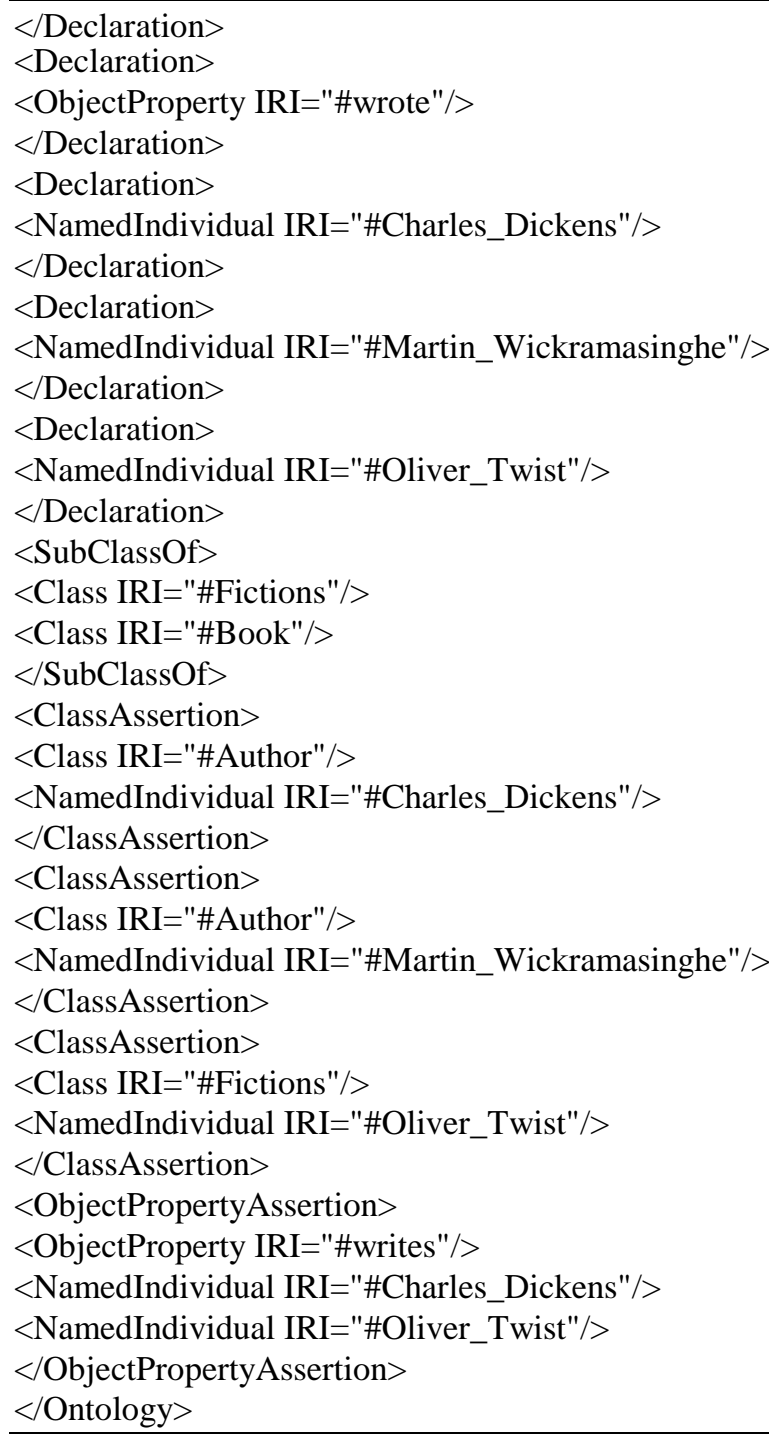

$</$ Declaration $>$

$<$ Declaration $>$

$<$ ObjectProperty IRI="\#wrote"/>

$</$ Declaration $>$

$<$ Declaration>

$<$ NamedIndividual IRI="\#Charles_Dickens"/>

$</$ Declaration $>$

$<$ Declaration $>$

$<$ NamedIndividual IRI="\#Martin_Wickramasinghe"/>

$</$ Declaration $>$

$<$ Declaration $>$

$<$ NamedIndividual IRI="\#Oliver_Twist"/>

$<$ /Declaration $>$

$<$ SubClassOf $>$

$<$ Class IRI="\#Fictions"/>

$<$ Class IRI="\#Book"/>

$<$ /SubClassOf $>$

$<$ ClassAssertion>

$<$ Class IRI="\#Author"/>

$<$ NamedIndividual IRI="\#Charles_Dickens"/>

$</$ ClassAssertion>

$<$ ClassAssertion>

$<$ Class IRI="\#Author"/>

$<$ NamedIndividual IRI="\#Martin_Wickramasinghe"/>

$</$ ClassAssertion $>$

$<$ ClassAssertion>

$<$ Class IRI="\#Fictions"/>

$<$ NamedIndividual IRI="\#Oliver_Twist"/>

$</$ ClassAssertion $>$

$<$ ObjectPropertyAssertion>

$<$ ObjectProperty IRI="\#writes"/>

$<$ NamedIndividual IRI="\#Charles_Dickens"/>

$<$ NamedIndividual IRI="\#Oliver_Twist"/>

$</$ ObjectPropertyAssertion $>$

$</$ Ontology $>$

The user can add a new concept to the system. The concept will be tokenized and classes, sub classes, instances, properties and relationships are identified.

"Martin Wickaramasinghe wrote fictions and short stories. Madolduwa is a fiction written by Martin Wickramasinghe."

Then the system updates its knowledge by extracting the relevant concepts or adding totally new contents.

\section{Updated ontology}

$<$ SubClassOf $>$

$<$ Class IRI="\#Fictions"/>

$<$ Class IRI ="\#Book"/>

4SubClassOf>

¿SubClassOf>

$<$ Class IRI="\#Short_story"/>

$<$ Class IRI="\#Book"i>

$</$ SubClassOf $>$

$<$ SubClassOf $>$

$<$ Class IRI="\#Subject_Specific"/>

$<$ Class IRI="\#Book"/>

$<$ SubClassef $>$

<ClassAssertion>

〈Class IRI="\#Fictions"/>

$<$ Namedindividual IRI="\#Madolduwa"/> 


\section{$</$ ClassAssertion $>$}

$<$ ClassAssertion>

$<$ Class IRI="\#Author"/>

$<$ NamedIndividual IRI="\#Martin_Wickramasinghe"/>

$</$ ClassAssertion $>$

$<$ ClassAssertion>

$<$ Class IRI="\#Fictions"/>

$<$ NamedIndividual IRI="\#Oliver_Twist"/>

$</$ ClassAssertion>

$<$ AnnotationAssertion $>$

$<$ AnnotationProperty abbreviatedIRI="rdfs:comment"/> $\langle$ IRI $>$ \#Martin_Wickramasinghe $</$ IRI $>$

IRI $>$ \#Madolduwa</IRI>

$</$ AnnotationAssertion $>$

$</$ Ontology $>$

\section{Evaluation}

Ontology Driven Continuous Learning Approach consists of two main research components and evaluation is done by considering both research components. Main research components are:

1) Ontology construction from the concept

2) Update the knowledge by integrating main ontology and new ontology.

In the ontology construction process, the accuracy is evaluated by considering three facts called identifying classes, instances and properties correctly. Statistical analysis is based on thirty different sentences belong to different complexity levels.

\section{Evaluate the accuracy of class identification}

H0: Accuracy of identifying classes by the ontology driven continuous learning approach is greater than or equal to 0.6

H1 : Otherwise

$$
\begin{gathered}
Z=\frac{\tilde{p}-p}{\sqrt{\frac{\tilde{p}(1-\tilde{p}}{n}}} \sim N(0,1) \\
=\frac{0.63-0.6}{\sqrt{\frac{0.63 \times(1-0.63)}{30}}} \sim N(0,1) \\
=0.34
\end{gathered}
$$

Since $p$ value $>0.05$ it does not reject $\mathrm{H} 0$

Therefore accuracy identifying classes by the ontology driven continuous learning approach is greater than or equal to 0.6 .

Similarly the accuracy property identification and instance identification are evaluated. Property identification and instance identification was equivalent to 0.6.

In the ontology construction process, the accuracy is evaluated by considering three facts called identifying classes, instances and properties correctly.

Statistical analysis is based on thirty different sentences belong to different complexity levels.

Knowledge updating component is also evaluated by considering the correct integration of classes, instances and properties. Statistical analysis is based on thirty different ontologies belong to different complexity levels. Further accuracy is evaluated by considering three different identification mediums and statistical analysis results are

\begin{tabular}{|c|c|c|c|c|}
\hline \multirow[t]{2}{*}{$\begin{array}{l}\text { Identification } \\
\text { Medium }\end{array}$} & \multirow[t]{2}{*}{$\begin{array}{l}\text { Type of } \\
\text { concept }\end{array}$} & \multicolumn{2}{|c|}{$\begin{array}{l}\text { Accuracy of } \\
\text { Identification }\end{array}$} & \multirow[t]{2}{*}{ Total } \\
\hline & & $\begin{array}{l}\text { Correctly } \\
\text { Identified }\end{array}$ & $\begin{array}{l}\text { Not } \\
\text { correctly } \\
\text { Identified } \\
\end{array}$ & \\
\hline \multirow[t]{4}{*}{ Human } & & & & \\
\hline & Class & $9.3 \%$ & $90.7 \%$ & $100.0 \%$ \\
\hline & Property & $10.5 \%$ & $89.5 \%$ & $100.0 \%$ \\
\hline & Instance & $13.3 \%$ & $86.7 \%$ & $100.0 \%$ \\
\hline \multirow{3}{*}{$\begin{array}{l}\text { Semi-Automated } \\
\text { (Human }+ \\
\text { Computer) }\end{array}$} & Class & $7.6 \%$ & $92.4 \%$ & $100.0 \%$ \\
\hline & Property & $8.7 \%$ & $91.3 \%$ & $100.0 \%$ \\
\hline & Instance & $11.3 \%$ & $88.7 \%$ & $100.0 \%$ \\
\hline \multirow[t]{3}{*}{ System } & Class & $21.8 \%$ & $78.2 \%$ & $100.0 \%$ \\
\hline & Property & $28.4 \%$ & $71.6 \%$ & $100.0 \%$ \\
\hline & Instance & $35.2 \%$ & $64.8 \%$ & $100.0 \%$ \\
\hline
\end{tabular}
shown in Table I.

TABLE I: Statistical ANALysis Table to MEASURE THE ACCURACy

The system was successfully developed with all mentioned capabilities and the research components. The ontology construction mechanism introduced in the system was designed with over $60 \%$ of expected accuracy and knowledge integration can be evaluated over $75 \%$ accuracy.

\section{CONCLUSION}

Human knowledge expands similar to the expansion of branches of a tree and when a person acquires new information, branches will be grown along the relevant concept nodes. Most of them have not focused on manipulating the existing knowledge similar to human learning. Information available in different formats cannot be understood by a computer or a machine due to lack of a proper knowledge representation mechanism. It always requires more human effort in feeding the knowledge to the computers or the knowledgebase.

The research 'Ontology Driven Continuous Learning Approach' opens up a new era in mankind by introducing an automated learning approach which can construct the knowledge by converting concepts into ontology and update the knowledge by integrating new concepts with the main ontology. The approach directly supports to two main learning strategies called rote learning and meaningful learning. Rote learning is memorizing information so that it can be recalled by the learner exactly the way it was read or heard.

There were few recognized challenges during the research process. Still people have the doubt on 'Can technology replace human intelligence?' and it was a huge challenge to find out supporting related work and technology. Constructing ontology from the concept can be defined as the major challenge of the research. Understanding the natural language concept by removing ambiguities was one of the major research component associated with the system. Identification of necessary classes, instances and properties 
by grasping the semantics of the concept was a really challenging task.

The system achieves the objectives of the research by presenting ontology based solution to represent natural language concepts into meaningful ontology and start the learning with minimal knowledge.

Eventually it expands the knowledge without human involvement. The software is endowed with inventions involving in knowledge representation via ontology, which are still under research. Knowledge extraction, knowledge comparison and knowledge integration can be defined as three main functions of the system. This system can be used as the main learning process of any application and the knowledge will be easily manipulated in any intelligence system by embedding this system.

It is important to totally simulate the human learning by constructing ontology for a concept which contains any level of complexity. Increase the complexity of the concept by raising the number of sentences and comprehensive level can be declared as an extension to the research 'Ontology Driven Continuous Learning Approach'.

\section{REFERENCES}

[1] E. A. Feigenbaum, "Themes and case studies of knowledge engineering," in D. Michie, ed., Expert Systems in the Micro-Electronic Age, Edinburgh University Press, Edinburgh, Scotland, 1979,

[2] E. Blomqvist, "OntoCase - A pattern-based ontology construction approach," On the Move to Meaningful Internet Systems 2007: CoopIS $D O A, O D B A S E$, GADA, and IS, pp. 971-988, Heidelberg: Springer, Berlin, 2007

[3] E. Ovchinnikova and K.-U. Kühnberger, "Aspects of automatic ontology extension: Adapting and re-generalizing dynamic updates," in Proc. the second Australasian Workshop on Advances in Ontologies, vol. 72, pp. 51-60, Australian Computer Society, Inc., Darlinghurst, Australia.

[4] G. Varelas, E. Voutsakis, and P. Raftopoulou, "Semantic similarity methods in WordNet and their application to information retrieval on the web," WIDM'05, November 5, 2005, Bremen, Germany.

[5] P. Buitelaar, D. Olejnik, and M. Sintek, "A protege plug-in for ontology extraction from text based on linguisitc analysis," presented at Semantic Web Symposium ESWS-2004, Greece, 2004.
[6] A. Maedche and S. Staab. TEXT-TO-ONTO Ontology Learning Environment. [Online]. Available: www.uni-koblenz.de/ staab/.../iccs2000softwaredemo_texttoonto

[7] M. Y. Dahab, H. A. Hassan, and A. Rafea, "TextOntoEx: Automatic ontology construction from natural English text," presented at 06th Artificial Intelligence and machine learning International Conference, Egypt. 2006

[8] T. Gruber, Ontology, Springer-Verlag, 2009.

[9] S. Grimm, P. Hitzler, and A. Abecker, "Knowledge representation and ontologies," Semantic Web Services, pp. 51-105, 2007.

[10] T. Gruber, "Toward principles for the design of ontologies used for knowledge sharing," International Journal Human-Computer Studies, pp. 4-5.

[11] What Is an Ontology, Lecture Notes University of Stanford.

[12] D. L. McGuinnes, "Ontologies come of age," in D. Fensel, J. Hendler, H. Lieberman, and W. Wahlster, ed., Spinning the Semantic Web: Bringing the World Wide Web to Its Full Potential, MIT Press, 2002.

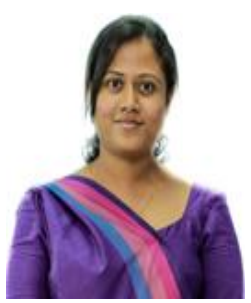

Dakshi T. K. Geeganage was born in Colombo, $\mathrm{Sr}$ Lanka in 1986. Tharanga received her BSc (Hons) in information technology with a first class in 2010 from Sri Lanka Institute of Information Technology, Malabe, Sri Lanaka. Tharanga completed the MSc in artificial intelligence at University of Moratuwa, Sri Lanka.

In January 2011, she joined the Department of Information Technology of Sri Lanka Institute of Information Technology as an instructor, and in June 2011, she became an assistant lecturer. Her current research interests include artificial intelligence, knowledge representation, ontology modelling and semantic web. She has several publications in the 8th International Conference on Computer Science and Education (ICCSE 2013), 2nd research symposium University of Uva Wellassa Sri Lanka. December 2011, 30th Anniversary International Conference of Open University of Sri Lanka, August 2010 and 6th research symposium, Sri Lanka Institute of Information Technology.

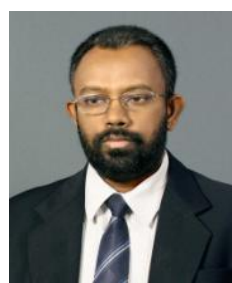

Asoka S. Karunananda is a senior professor of Information Technology University of Motratuwa, Sri Lanka. At present he is the dean of Research and Development of General Sir John Kotelawala Defence University. His research interests include multi agent systems, ontological modelling, machine translation, mindfulness, and theory of computing. He has more than 100 international publications to 